\title{
Integrated analysis of immune-related genes in endometrial carcinoma
}

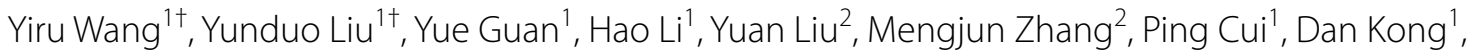 \\ Xiuwei Chen ${ }^{1 *}$ (D) and Hang Yin ${ }^{2^{*}}$
}

\begin{abstract}
Background: Exploring novel and sensitive targets is urgent due to the high morbidity of endometrial cancer (EC). The purpose of our study was to explore the transcription factors and immune-related genes in EC and further identify immune-based IncRNA signature as biomarker for predicting survival prognosis.

Methods: Transcription factors, aberrantly expressed immune-related genes and immune-related IncRNAs were explored through bioinformatics analysis. Cox regression and the least absolute shrinkage and selection operator (LASSO) analysis were conducted to identify the immune and overall survival (OS) related IncRNAs. The accuracy of model was evaluated by Kaplan-Meier method and receiver operating characteristic (ROC) analysis, and the independent prognostic indicator was identified with Cox analysis. Quantitative real-time polymerase chain reaction (qRTP(R) were conducted to detect the accuracy of our results.

Results: A network of 29 transcription factors and 17 immune-related genes was constructed. Furthermore, four immune-prognosis-related IncRNAs were screened out. Kaplan-Meier survival analysis and time-dependent ROC analysis revealed a satisfactory predictive potential of the 4-IncRNA model. Consistency was achieved among the results from the training set, testing set and entire cohort. The distributed patterns between the high- and low-risk groups could be distinguished in principal component analysis. Comparisons of the risk score and clinical factors confirmed the four-IncRNA-based signature as an independent prognostic indicator. Last, the reliability of the results was verified by qRT-PCR in 29 cases of endometrial carcinoma and in cells.
\end{abstract}

Conclusions: Overall, our study constructed a network of transcription factors and immune-related genes and explored a four immune-related IncRNA signature that could serve as a novel potential biomarker of EC.

Keywords: Long non-coding RNA, Immune, Biomarkers, Transcription factor, Endometrial cancer, The cancer genome atlas

\section{Background}

Endometrial cancer (EC) is considered as the most common gynecological malignancy [1]. The incidence and mortality of EC have shown an upward tendency annually worldwide, affecting approximately 63,000 new

\footnotetext{
*Correspondence: Chenxiuwei1023@163.com; yinhangwin@163.com ${ }^{\dagger}$ Yiru Wang and Yunduo Liu contributed equally to this study

${ }^{1}$ The Department of Gynecologic Oncology, Harbin Medical University Cancer Hospital, Harbin 150040, Heilongjiang, China

${ }^{2}$ The Department of Radiotherapy Oncology, Harbin Medical University Cancer Hospital, Harbin, Heilongjiang, China
}

patients and contributing to over 11,000 deaths in the U.S. each year [2]. The increasing morbidity of EC in the United States and worldwide is particularly due to obesity, senility, early menarche, late menopause and Lynch syndrome [3]. Endometrial cancer cells are of glandular epithelial origin and are invasive [1]. Patients with lowdegree, non-invasive and early-stage tumors frequently have a comparatively favorable prognosis [4]. In contrast, patients with advanced EC with high-grade histologic subtypes have worse survival $[5,6]$. 
Therefore, strengthening the mechanism research of $\mathrm{EC}$ is of great clinical significance.

Long noncoding RNAs (lncRNAs) are longer than $200 \mathrm{nt}$ in length and have no protein coding functions [7]. LncRNAs are a class of transcripts involved in the regulation of signal pathway activities by influencing proteinencoding gene expression [8]. Overwhelming evidence suggests that lncRNAs regulate gene expression by means of chromatin modification, transcriptional activation and transcriptional interference in the occurrence and development of tumors [9]. In addition, based on multiple relevant studies, some lncRNAs have been proven as biomarkers for early diagnosis and prognosis evaluation for their apparent cell and tissue-type specificity [10]. As our previous study found, novel 11-lncRNA have been identified as the prognostic factor of head and neck squamous cell carcinoma [11], and a 9-IncRNA signature has been suggested as an independent prognostic indicator to predict survival in clear cell renal cell carcinoma [12]. Nevertheless, studies of lncRNAs in EC remain rare. Therefore, exploring key EC-related lncRNAs to predict survival and systematically clarifying their functions and clinical significance in EC are indispensable.

The immune system plays a significant role in the process of tumorigenesis, tumor development and metastasis [13]. In the last 3 years, the clinical application of immunotherapies in oncology has gained considerable attention showing promising progress. To date, immunotherapy with checkpoint inhibitors has been demonstrated to have the ability to improve gynecologic cancer clinical trial outcomes [14]. For instance, the positive modulation of adaptive immunity through anti-PD-1 or anti-PD-L1 antibodies promotes $\mathrm{T}$ cell proliferation, enhances the anti-tumor effect of $\mathrm{T}$ cells and represses the immune escape of cancer cells, ultimately resulting in gynecological tumor regression [15]. Most previous researches have concentrated on the function of proteins in this progression, but further research on the specific functions of RNAs is still relatively scant [16]. LncRNAs are emerging as regulatory complexes that influence gene expression and pathways in the modulation of the immune system [17]. In addition, transcription factors (TFs) have been reported to trigger dynamic changes in immune cells to a certain degree and participate in the regulation of the immune response [18, 19].

Nevertheless, the clinical implication of immunerelated lncRNAs in endometrial cancer prognostication has not been well investigated. Hence, it is crucial to confirm the complete landscape of lncRNAs that are engaged in the regulation of the immune response. LncRNAs in the context of the immune system may be of great significance not only in providing reasonable methods for immunotherapy but also in offering accurate therapeutic options for tumors.

In the current study, we explored transcription factors and immune-related genes from the TCGA database. Then, immune-related IncRNAs were explored. Further, we found the four-lncRNA could predict survival and serve as biomarker. Kaplan-Meier survival analysis and time-dependent ROC analysis revealed the satisfactory predictive potential of the model. Consistency was achieved among the results from the training set, testing set and entire cohort. The distributed patterns between the high- and low-risk groups can be distinguished in principal component analysis. Next, comparisons of the risk score and clinical factors confirmed the fourlncRNA-based signature as an independent prognostic indicator. We compared the accuracy of our signature with other existing predictive models, and explored the four lncRNAs separately in database. Finally, we validated gene expression levels in clinical samples and cell lines by qRT-PCR.

\section{Methods \\ Data collection and differential expression analysis}

The clinical data including age, stage and survival status were obtained from The Cancer Genome Atlas (TCGA) database (https://portal.gdc.cancer.gov/). Patients whose clinical characteristic information was incomplete were excluded. In total, 541 endometrial cancer patients were enrolled in our study. The gene data were derived from the TCGA. The gene sequence data of $552 \mathrm{EC}$ tumor tissues and 23 normal tissues were collected. And Differential expression (DE) analysis of genes was carried out by $\mathrm{R}$ software limma package. In our research, the criteria for identifying differentially expressed genes were $|\log 2 \mathrm{FC}|>1$ and FDR $<0.05$ (FC, fold change; FDR, false discovery rate).

\section{Differentially expressed transcription factors and immune genes}

Data on 318 transcription factors (TFs) was obtained from Cistrome (https://cistrome.org/) [20], and the list of 2498 immune genes was downloaded from ImmPort (https://immport.niaid.nih.gov) [21]. Next, differentially expressed TFs were extracted from the overlap between TFs and all of differentially expressed genes (DEGs). And we acquired the immune-related DEGs in the same way. Then, the immune-prognosis-associated DEGs were screened by using univariate Cox regression model of immune-related DEGs. The Cox regression analysis subjected was conducted in survival package of R. Cytoscape 3.7.2 was applied to visualize the interaction network of TFs and immune-OS-related DEGs [22]. 


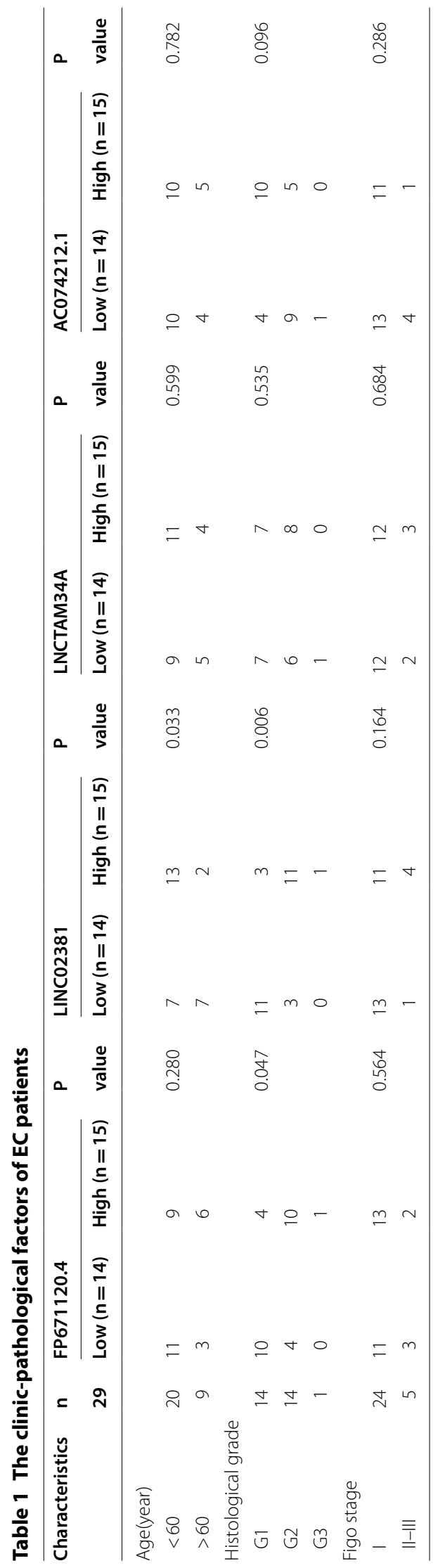




\section{Identification of immune-related IncRNAs}

The co-expression analysis can deeply reflect the expression regulation relationship between genes. We obtained the immune-related lncRNA associated with prognosis by co-expression analysis of immune-OS-related DEGs and lncRNAs. And the correlation analysis was conducted by the limma package for R. According to the analysis results, immune-related lncRNAs ( $\mid$ cor $\mid>0.4$, $\mathrm{P}<0.001$ ) were screened for follow-up study.

\section{Functional enrichment analysis}

We explored the Gene Ontology (GO) and KEGG pathways with the clusterProfiler package to elucidate the molecular functions and cellular components of the differentially expressed genes.

\section{Identification of prognostic IncRNAs}

All 541 patients were randomly divided into a training set (272 patients) and a testing set (269 patients). Then, univariate and multivariate Cox regression analyses were performed. The risk score was an indicator of predicting an EC patient's prognostic risk and that of each individual sample was computed with the formula shown in Additional file 1: Figure S1. All individuals were divided into high- and low-risk groups according to differentially expressed genes in the high- or low-risk score. The overall survival (OS) in the high- and lowrisk groups was compared by Kaplan-Meier survival curves. Receiver operating characteristic (ROC) analysis was used to assess the accuracy and diagnostic value of the lncRNA risk model.

\section{Cell culture}

The normal human endometrial epithelial cell line hEEC and human endometrial adenocarcinoma cell lines HEC-1A and Ishikawa were obtained from Heilongjiang Cancer Institute (Harbin, China). All cell lines were cultured in Dulbeco's modified eagle medium (DMEM) supplemented with $10 \%$ fetal bovine serum (FBS) and maintained at $37^{\circ} \mathrm{C}$ with $5 \% \mathrm{CO} 2$.

\section{Collecting EC samples and performing quantitative} real-time polymerase chain reaction (qRT-PCR) verification After obtaining the approval of the ethics committee of Harbin Medical University, we prospectively collected 29 pairs of endometrial cancer tissues and normal tissues from patients in the Gynecology Department of the Cancer Hospital of Harbin Medical University, between January 2019 to December 2019. All the participants signed informed consent forms (Additional file 2: Figure S2). All patients were diagnosed with primary endometrial cancer and did not receive adjuvant or neoadjuvant therapy before surgery, but patients with high-risk factors received postoperative radiotherapy. The characteristics of patients were listed in Table 1. All tissue specimens were quickly frozen in liquid nitrogen and stored at $-80^{\circ} \mathrm{C}$. Total RNA was extracted from the sample using TRIzol reagent (Invitrogen, Carlsbad, CA), and then reverse transcription and PCR reactions were performed using ReverTra Ace qPCR RT-PCR kit (Toyobo, Shanghai). All RNA-primers were obtained from Generay Biotech (Shanghai, China). The sequences of applied primers were listed in Additional file 3: Table S1. Results were normalized to $\beta$-actin and calculated through $2^{-\Delta \Delta C t}$ method. The detailed information of this experiment was the same as our previous study [23,24], and all steps were carried out according to the manufacturer's instructions.

\section{Statistical analysis}

The differentially expressed genes between tumor and normal tissues were analysed by $\mathrm{R}$ software package "limma". Cox correlation analysis was used to confirm the relationships between the selected 4-IncRNA and patient outcomes. Survival curves were drawn with the survival package for R. The distributed patterns of high- and lowrisk subsamples were described by carrying out principal component analysis (PCA). $\mathrm{P}<0.05$ was regarded to indicate statistically significant differences.

\section{Results}

The exploration of immune-related and differently expressed genes and TFs in EC

Here, we identified 6267 DE genes (3861 upregulated genes and 2406 downregulated genes) between tumor and normal tissues (Fig. 1a, b). Afterwards, we collected 2498 immune-related genes. The results of differential gene expression analysis showed that 410 immune-relevant genes were differentially expressed in EC (Fig. 1c, d). The prognostic association between the $410 \mathrm{DE}$ genes and patient OS was estimated by univariate Cox analysis, and 53 immuno-OS-related DE genes with a P-value $<0.05$ were distinguished for the following analysis (Additional file 4: Figure S3). A total of 102 TFs (55 upregulated and 47 downregulated) were identified as differentially expressed between tumor and normal samples and were also included in the $6267 \mathrm{DE}$ genes (Fig. 1e, f).

\section{The network of TFs and genes}

To enhance the understanding of the potential function of DE TFs and prognosis-related DE immune genes in EC, we constructed a TF and gene network based on the coexpression method. The interaction relationships are listed in Additional file 5: Table S2. The interactional network is shown in Fig. 2. 

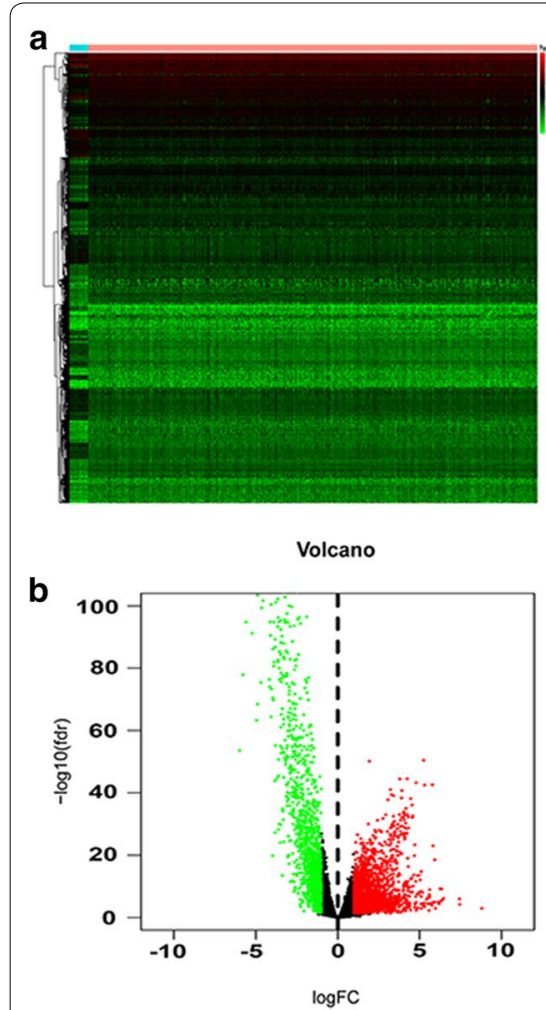

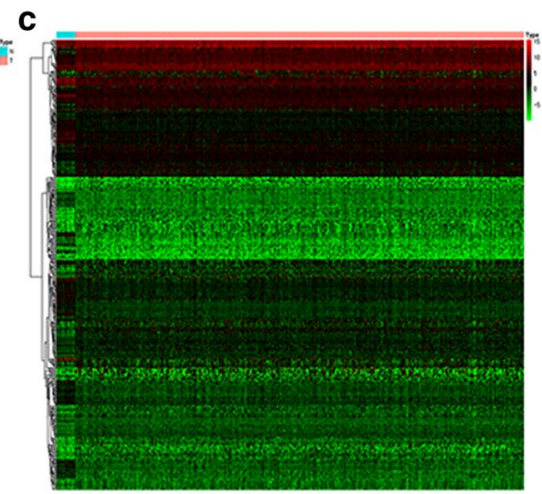

Volcano

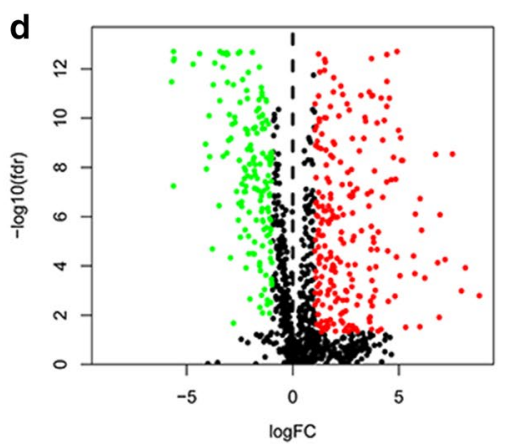

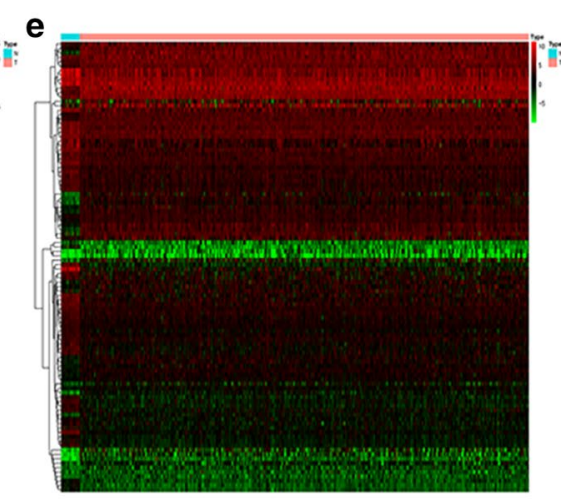

Volcano

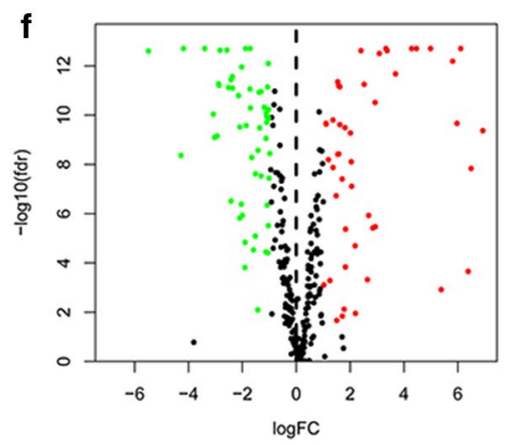

Fig. 1 Heatmap and volcano plot show the different expressions of genes between 552 tumor tissues and 23 normal controls in endometrial cancer (EC). The green or red plot represented down-or up-regulation of genes respectively. $\mathbf{a}, \mathbf{b}$ DE genes. $\mathbf{c}, \mathbf{d} D E$ immune-related genes. e, $\mathbf{f}$ DE transcription factors. DE differentially expressed

\section{GO and KEGG analysis of TFs and immune-related genes}

The study indicated that there were 29 GO terms and 30 enrichment pathways. Some tumor-related regulatory pathways were observed, including PD-L1 expression and PD-1 checkpoint pathway, MAPK pathway, PI3-Akt pathway and so on. The enrichment analysis results demonstrated the enrichment of regulatory functions, such as transcription factor complex, nuclear chromatin, and DNA-binding transcription activator activity. The GO term results in EC were shown in Fig. 3a, b, and the significantly enriched KEGG pathways in EC were shown in Fig. 3c, d.

\section{Identification of prognostic IncRNAs}

A total of 204 lncRNAs that may be related to immunity were explored. Then, we divided 541 patients into the training set and testing set by the complete randomization method. By univariate Cox regression analysis in the training set, 4 immune-associated lncRNAs were identified to correlate with OS $(\mathrm{P}<0.01)$. Then, a LASSO regression model was carried out to perform the next filtering of the 4 lncRNAs mentioned before. Glmnet from $\mathrm{R}$ software package was used for lasso regression analysis (iteration $=1000$ ). The trajectory changes of the coefficients of four independent variables were presented in Fig. 4a. Furthermore, cross-validation was applied for model construction, as shown in Fig. 4b, indicating that the mean cross-validated error was minimal when $\lambda=0.0216$. At this point, the 4 immune-related lncRNAs were confirmed to have close relativity with overall survival in EC. Afterwards, the result of multivariate Cox regression showed that among the four lncRNAs, three lncRNAs (FP671120.4, LINC02381 and AC074212.1) with positive coefficients may be poor prognostic indicators, while the remaining lncRNA(LNCTAM34A) could be a favorable prognostic factor (Fig. 4c).

\section{The 4-IncRNA signature for survival prediction}

In the training cohort, 272 samples were classified into a high-risk group $(\mathrm{n}=136)$ and a low-risk group $(n=136)$ according to the calculated median cutoff value of the risk score. The Kaplan-Meier survival curve analysis revealed that patients with high-risk scores had an obviously poorer OS than those with low-risk scores $(\mathrm{P}=4.602 \mathrm{e}-03$, Fig. 5a). The AUC for the 4-lncRNA signature achieved 0.717 (Fig. 5b). The distribution of the risk score, survival duration of EC patients and the expression profiles plotted by risk 


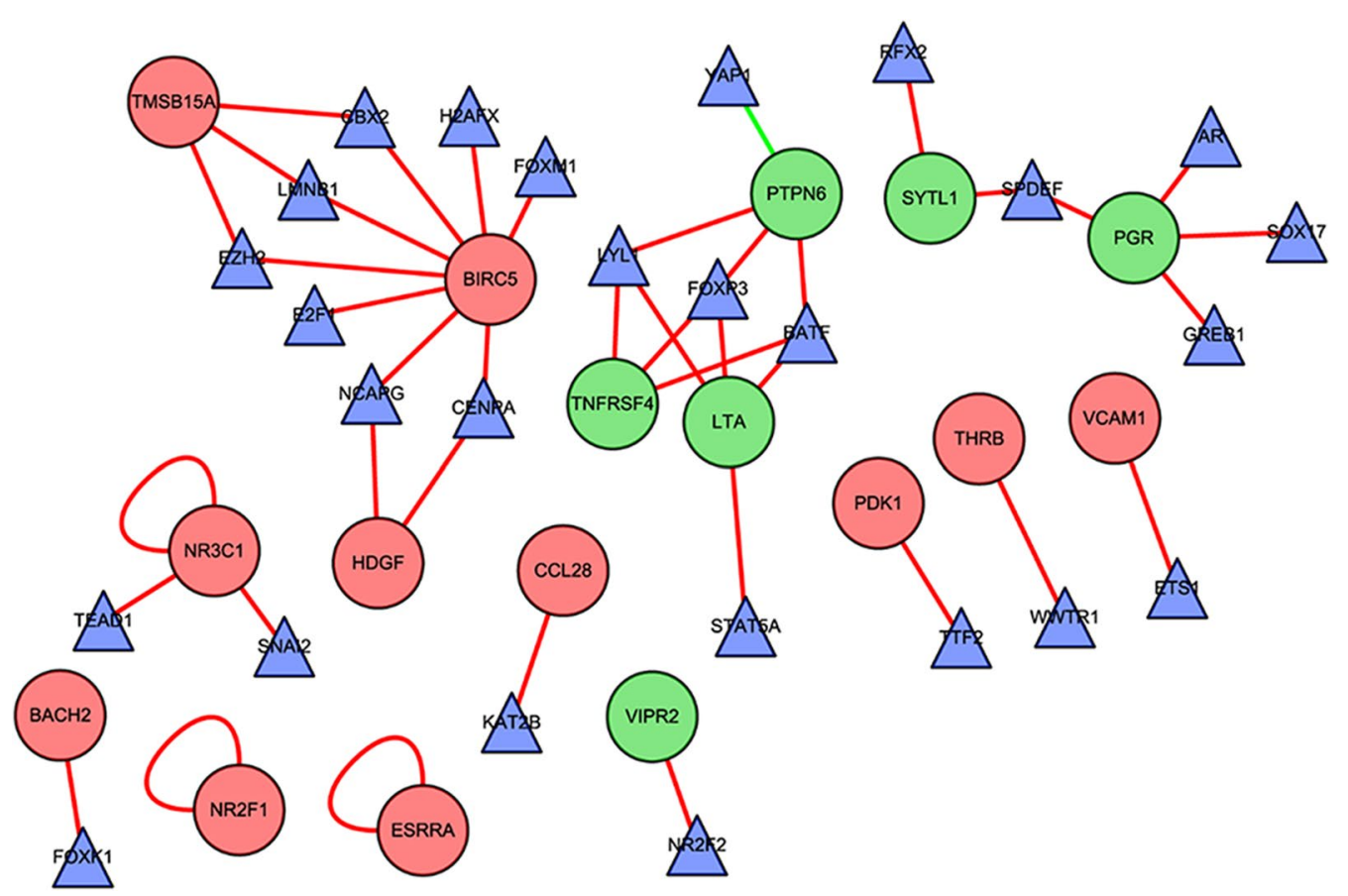

Fig. 2 The network of differentially expressed transcription factors and immune-OS-related genes. The red balls indicated the upregulated genes and the green balls indicated the downregulated genes associated with immune and survival. The triangles are differentially expressed transcription factors. Red or green line and semicircle indicated positive or negative correlation individually

heatmap of the 4 prognostic lncRNAs are demonstrated in Fig. 5c. The patients in the high-risk score group suffered poorer survival than patients in the low-risk score group.

The testing set and entire set were respectively divided into a high-risk group ( $\mathrm{n}=137$ in the testing set, $\mathrm{n}=273$ in the entire set) and a low-risk group $(n=132$ in the testing set, $n=268$ in the entire set) according to the expression of the 4-lncRNA. The results showed that patients with high risk scores had poorer survival outcomes than patients with low risk scores (Additional file 6: Figure S4a, Additional file 7: Figure S5a). The AUC for the 4-lncRNA signature in the testing set and the entire set reached 0.686 and 0.703 , respectively (Additional file 6: Figure S4b, Additional file 7: Figure S5b).

PCA was performed to detect the biological function of 4-lncRNA signature in EC, based on 4 lncRNAs in model, the whole genome expression set and immunerelated lncRNA set (Fig. 5d-f). By using four lncRNAs in the signature and immune related lncRNAs, patients in low- and high-risk groups were separated into two different directions. It indicated that EC patients in low- and high-risk groups generally displayed in distinct immune status patterns, and the different immune states can be distinguished by the lncRNA signature.

\section{Assessment of independent risk factors}

The estimation and verification of independent risk factors were conducted by Cox regression analyses. Univariate Cox regression analysis showed that age, histologic grade and risk score based on 4 immune-related lncRNA signature were identified as factors influencing survival (Fig. 6a). The multivariate Cox regression results demonstrated that the aforementioned features including age $(\mathrm{HR}=1.023, \mathrm{P}=0.049)$, grade $(\mathrm{HR}=2.378, \mathrm{P}<0.001)$ and risk score $(\mathrm{HR}=1.045, \mathrm{P}<0.001)$, were all independent prognostic indicators of EC (Fig. 6b). Moreover, ROC curves were calculated to explore the prognostic forecast capabilities and accuracy of the above factors. As Fig. $6 \mathrm{c}$ shown, the 4-lncRNA signature associated with immunity displayed a better AUC (AUC $=0.694)$ and can serve as an effective index to independently predict prognosis.

To evaluate the predictive ability of the signature and obtain more satisfactory results, survival analysis was performed in the randomly regrouped training set $(\mathrm{n}=272)$ and test set $(\mathrm{n}=269)$. As Additional file 8: Figure S6 and Additional file 9: Figure S7 shown, age $(P=0.005)$, grade $(P=0.002)$ and risk score $(P<0.001)$ were directly related to the prognosis of patients in training set. And in testing set, only grade $(\mathrm{P}=0.004)$ and risk score $(\mathrm{P}<0.001)$ were related to prognosis. Multivariate Cox regression analysis indicated that 


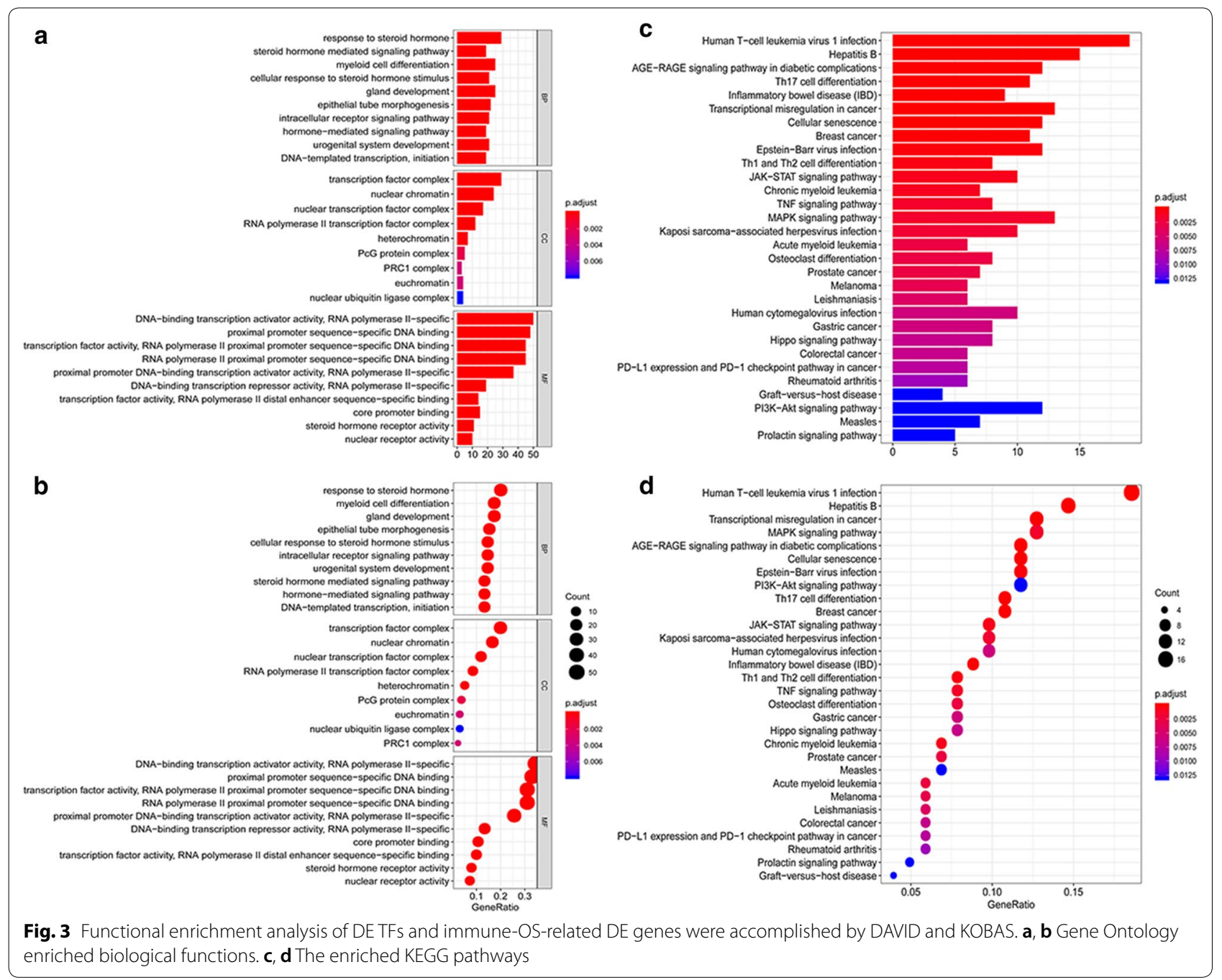

aside from age (training set: $\mathrm{P}=0.055$, testing set: $\mathrm{P}=0.177$ ), only grade (training set: $\mathrm{P}=0.004$, testing set: $\mathrm{P}=0.012$ ) and risk score (training set: $\mathrm{P}=0.002$, testing set: $\mathrm{P}<0.001)$ were statistically independent predictive indicators of endometrial cancer. The AUC for the risk score (training set: $\mathrm{AUC}=0.733$, testing set: $A U C=0.657$ ) based on 4-lncRNA signature in both training set and testing set was higher than that for grade (training set: $\mathrm{AUC}=0.665$, testing set: $\mathrm{AUC}=0.635$ ) and age (training set: $\mathrm{AUC}=0.651$, testing set: AUC $=0.544)$. It indicated that 4-lncRNA had the ability to compete sufficiently with traditional clinical factors to predict OS of EC patients. The results demonstrated the superiority of 4-lncRNA in predicting HCC patient OS compared with classical clinical and pathological staging systems.

\section{Comparison of the immune-related IncRNA signature with other prognostic models}

In order to determine whether this immune-related lncRNA signature had more superiority than other endometrial cancer prognostic biomarkers, we compared our signature with nine-gene signature [25], six-gene signature [26], seven-gene signature [27], and nine-gene signature [28]. The genes in these signatures were obtained from the literature, and we constructed the ROC curves and survival curves of the entire cohort. As shown on Fig. 7, the AUC values of OS in these models were 0.703 , $0.675,0.597,0.61$ and 0.665 , respectively. Through analysis and comparison of these signatures, we know that the accuracy of our signature in predicting prognosis of endometrial cancer is higher than that of other four biomarkers (Table 2). 

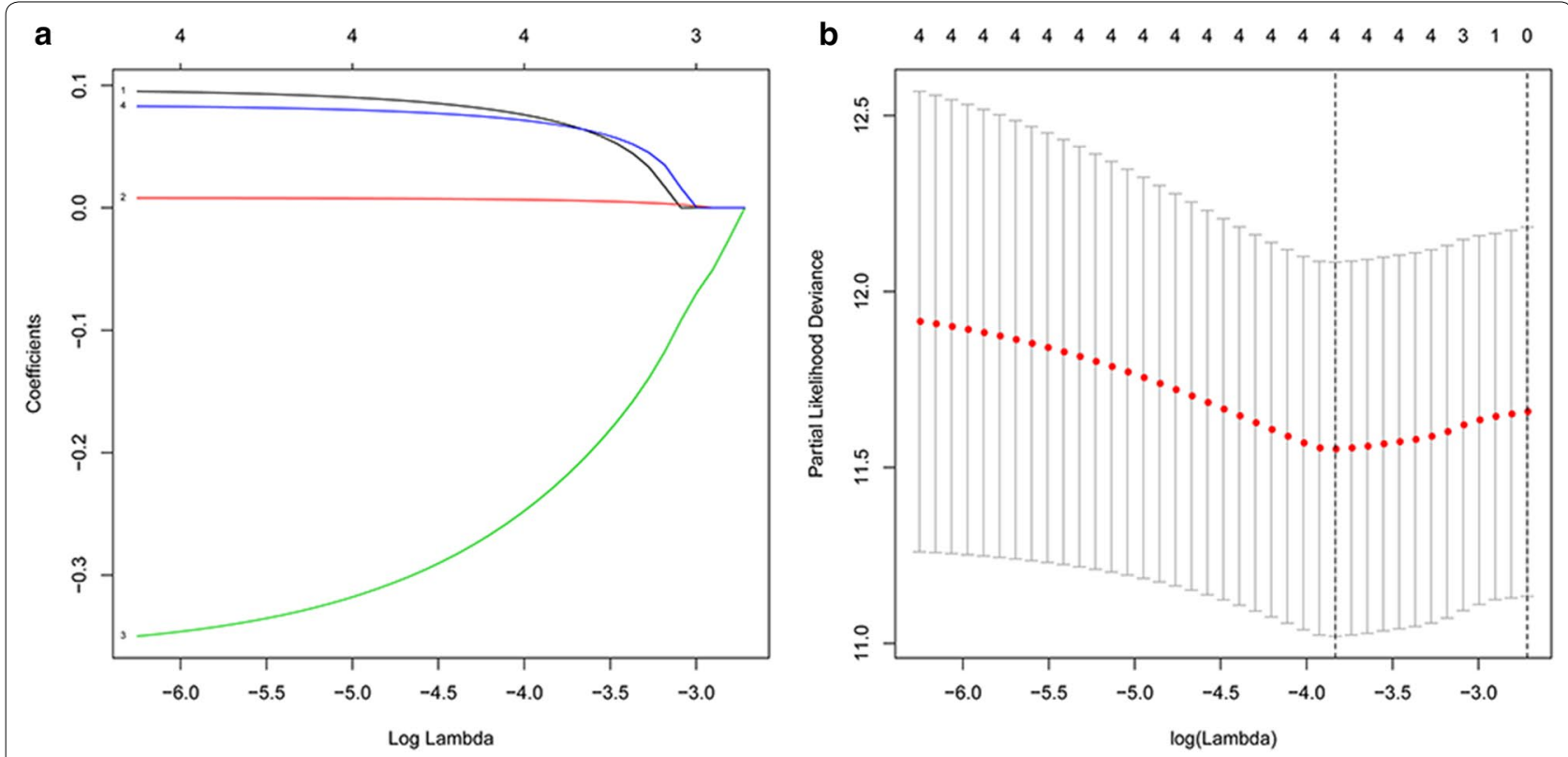

C

Hazard ratio

FP671120.4

$(N=272)$

$\left(1.03^{1.1}-1.18\right)$

LINC02381

$(N=272)$

$\left(1.00^{1.0}-1.02\right)$

0.102

$(N=272)$

$(0.52-0.92)$

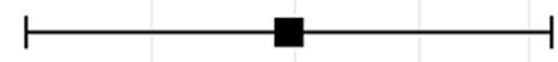

0.012 *

AC074212.1

$(N=272)$

$\left(1.03^{1.1}-1.14\right)$

\# Events: 40; Global p-value (Log-Rank): 0.0003579 AIC: 378.06; Concordance Index: 0.67

Fig. 4 Identification of immune related prognostic IncRNA. a, b Least absolute shrinkage and selection operator analysis (LASSO) parameter adjustment and lambda profiles of 4 IncRNA selected by univariate Cox regression analysis. c Multivariate Cox regression analysis of 4 IncRNA. IncRNA, long noncoding RNA 


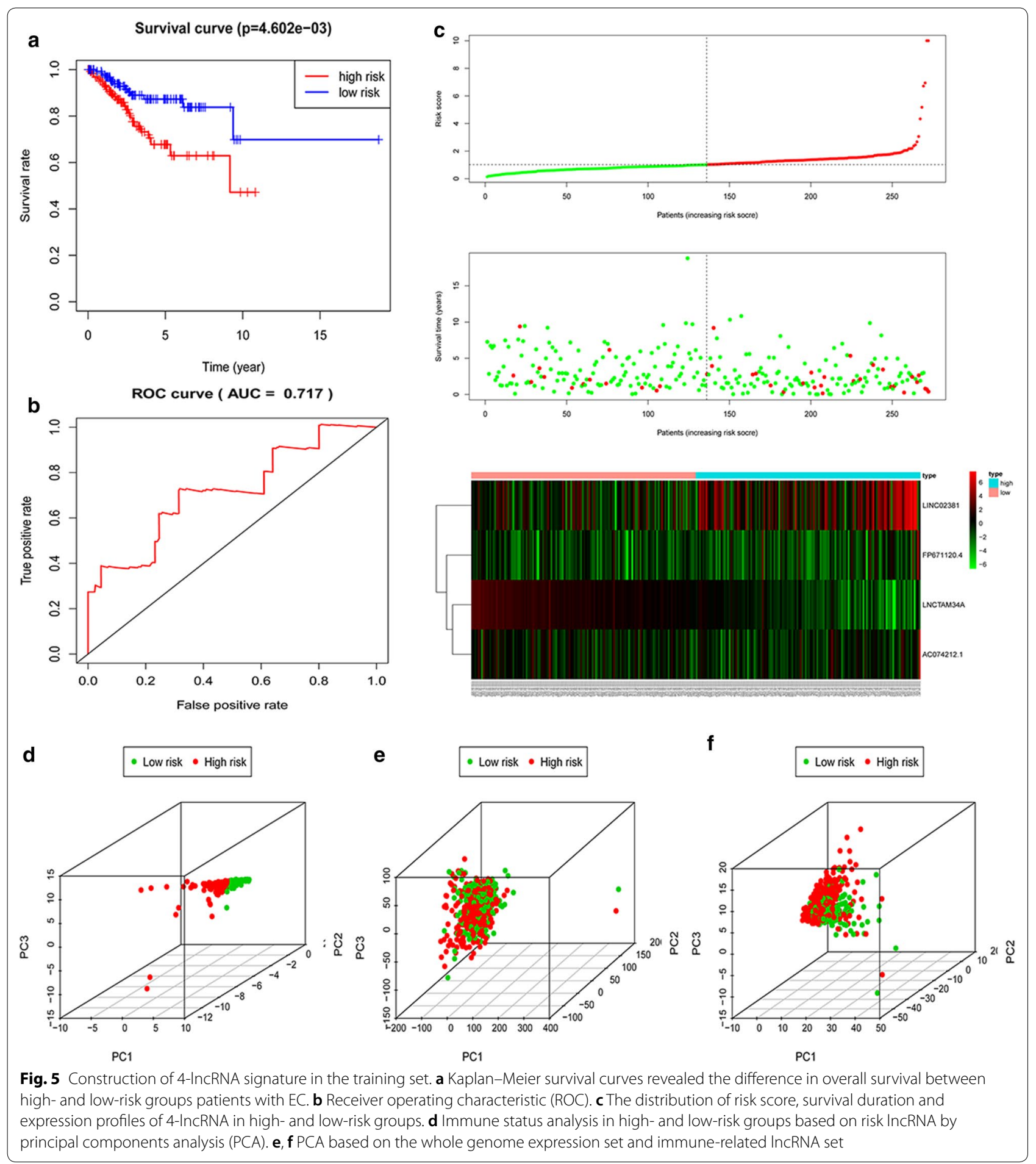

\section{Prognostic value of each of the four IncRNAs}

We compared the corresponding expression levels of each of the four lncRNAs (FP671120.4, LINC02381, LNCTAM34A and AC074212.1) between EC tissues and non-tumor tissues (Fig. 8). Finally, a total of 541 EC patients were divided into the high- and low-expression groups by utilizing the median expression level of each lncRNA as the critical value. Kaplan-Meier survival analysis was employed to explore the prognostic 


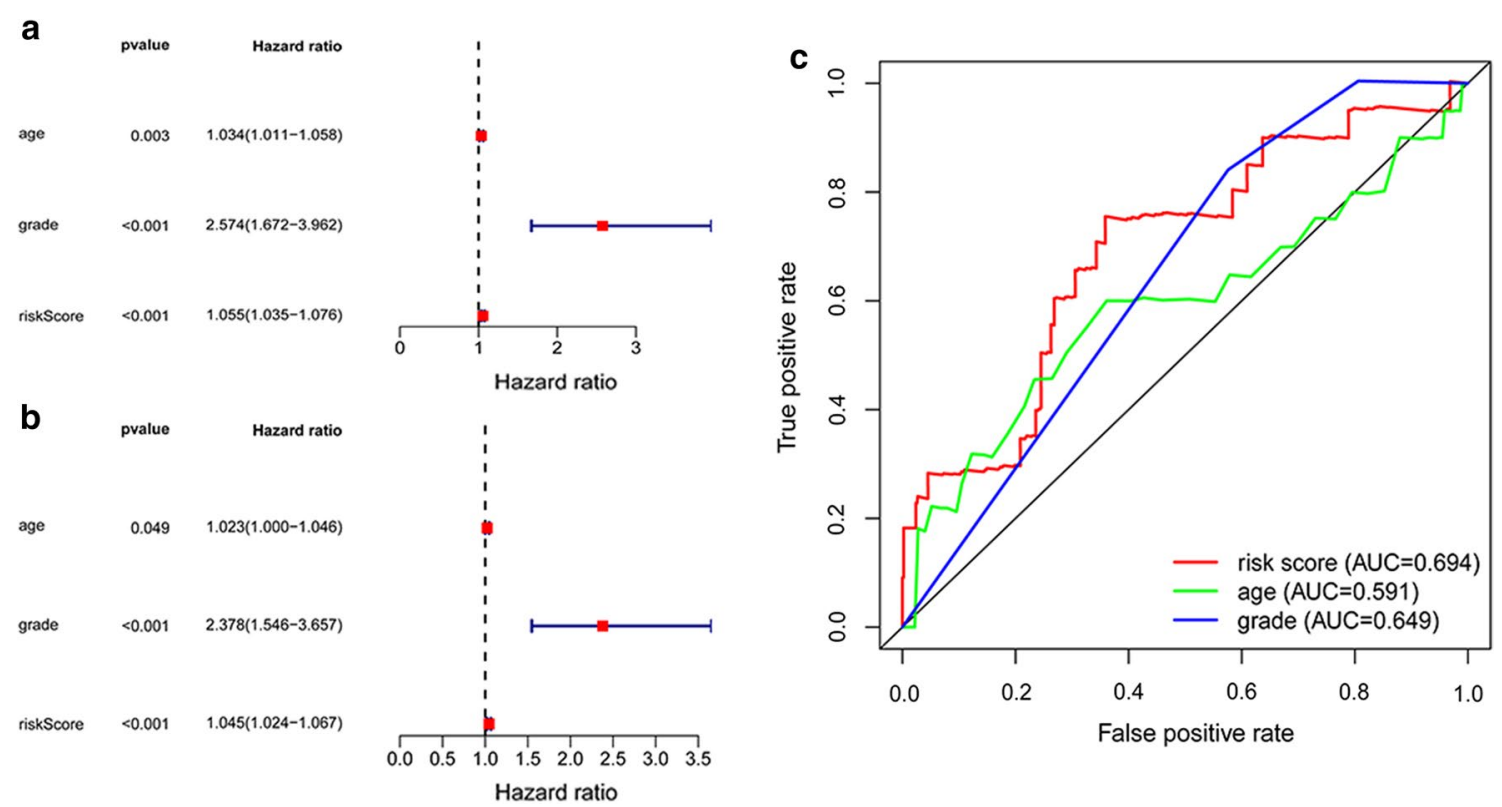

Fig. 6 Assessment of independent risk factors. a Age, grade and risk score were the independent prognostic indicators by univariate analysis. b Age, grade and risk score were the independent prognostic indicators by multivariate analysis. c ROC curves showed the predict potential of 4-InCRNA signature

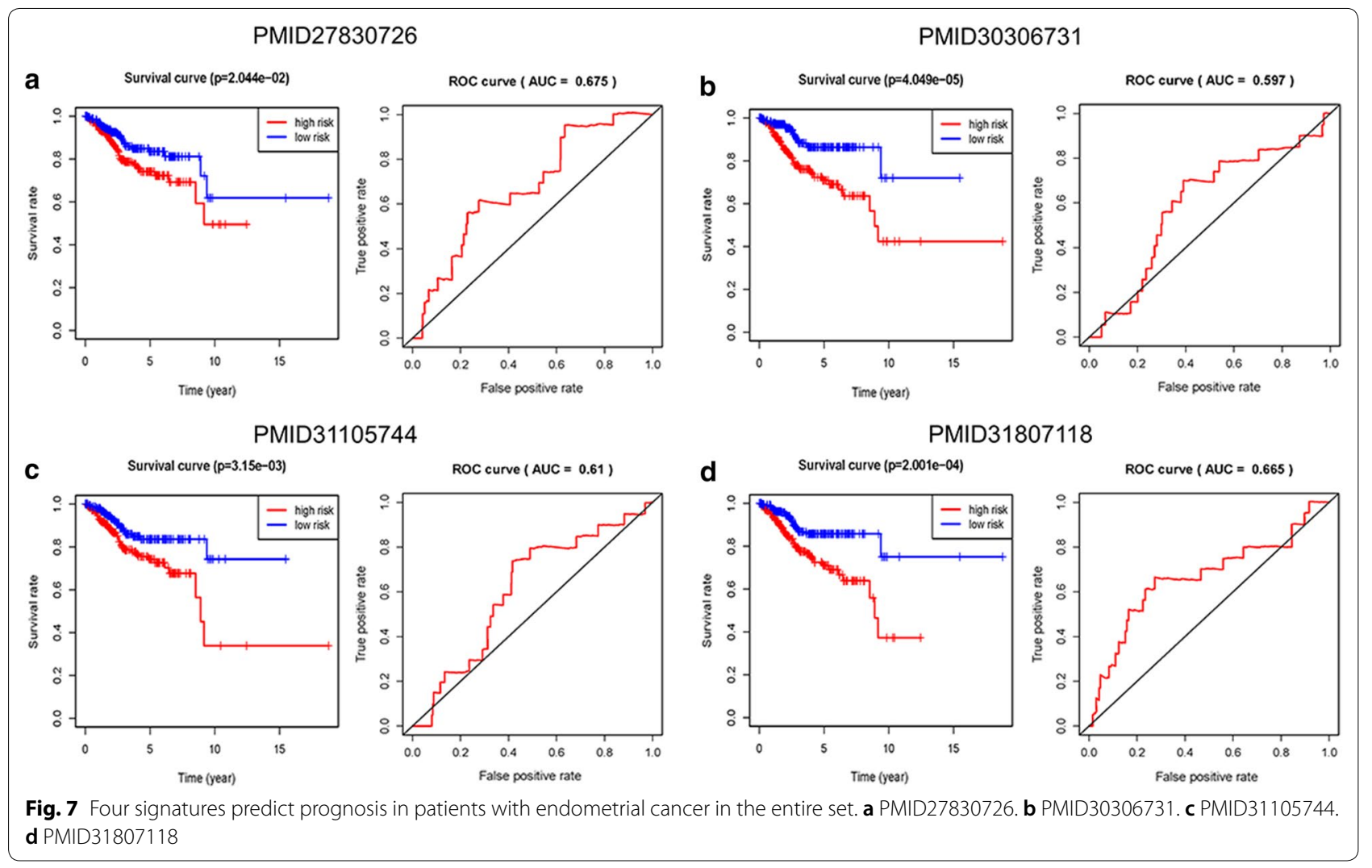


Table 2 Comparison of the immune-related IncRNA signature with four other prognostic models

\begin{tabular}{lll}
\hline Model & AUC & P-value \\
\hline Immune-related IncRNA signature & 0.703 & $6.902 \mathrm{e}-06$ \\
Nine-gene signature (PMID27830726) & 0.675 & $2.044 \mathrm{e}-02$ \\
Six-gene signature (PMID30306731) & 0.597 & $4.049 \mathrm{e}-05$ \\
Seven-gene signature (PMID31105744) & 0.61 & $3.15 \mathrm{e}-03$ \\
Nine-gene signature (PMID31807118) & 0.665 & $2.001 \mathrm{e}-04$ \\
\hline
\end{tabular}

capacity of each lncRNA, and the analysis results are presented in Fig. 9.

\section{QRT-PCR verification}

In order to further evaluate the reliability of the immunerelated signature, we measured the actual expression of four lncRNAs in the tissues of 29 patients by qRT-PCR. Compared with adjacent normal tissues, FP671120.4, LINC02381 and AC074212.1 were upregulated while LNCTAM34A were downregulated in EC tissues (Fig. 10). Similarly, compared with normal endometrial epithelial cell line hEEC, the expression level of FP671120.4 and LINC02381 significantly upregulated in EC cell lines (Fig. 11). In addition, AC074212.1 expression was upregulated and LNCTAM34A was downregulated in Ishikawa. However, the expression of AC074212.1 and LNCTAM34A were both no significant difference between HEC-1A and hEEC. The results of qRT-PCR verification in 29 patients with endometrial cancer and in cells were consistent with the above-mentioned bioinformatics results. It revealed the validity and reliability of the biological signature we constructed. The flowchart of our research strategy is described in Fig. 12.

\section{Discussion}

Endometrial cancer ranks as the fifth most common cause of cancer death in the United States, increasingly, threatening female patients' lives [29]. The discovery of objective and susceptible indicators is crucial to optimize clinical diagnosis and has instructive significance in judging the prognosis of EC accurately.
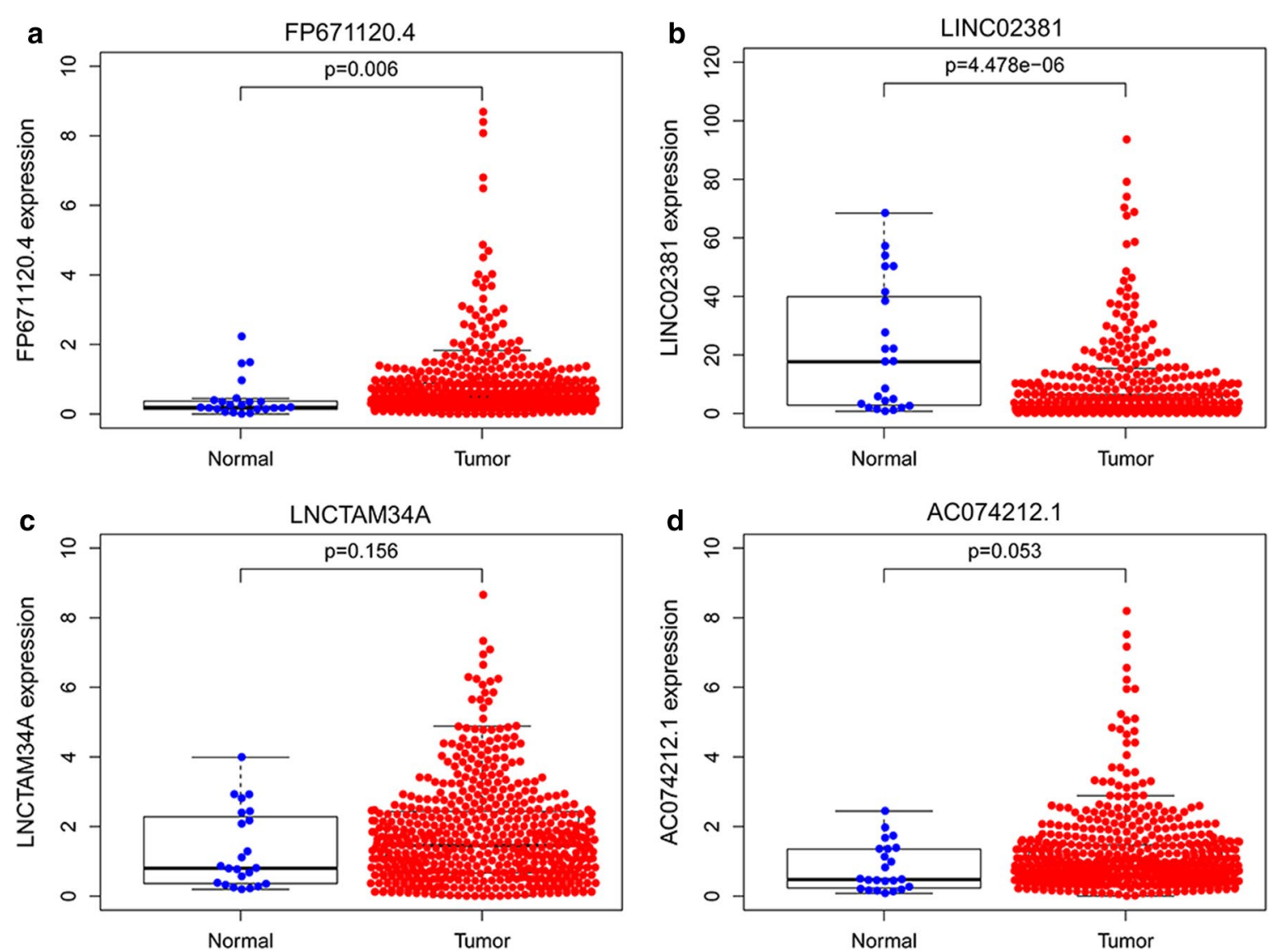

Fig. 8 The comparison of immune-related IncRNA expression between EC tissues and normal tissues. a FP671120.4. b LINC02381. c LNCTAM34A. d AC074212.1 

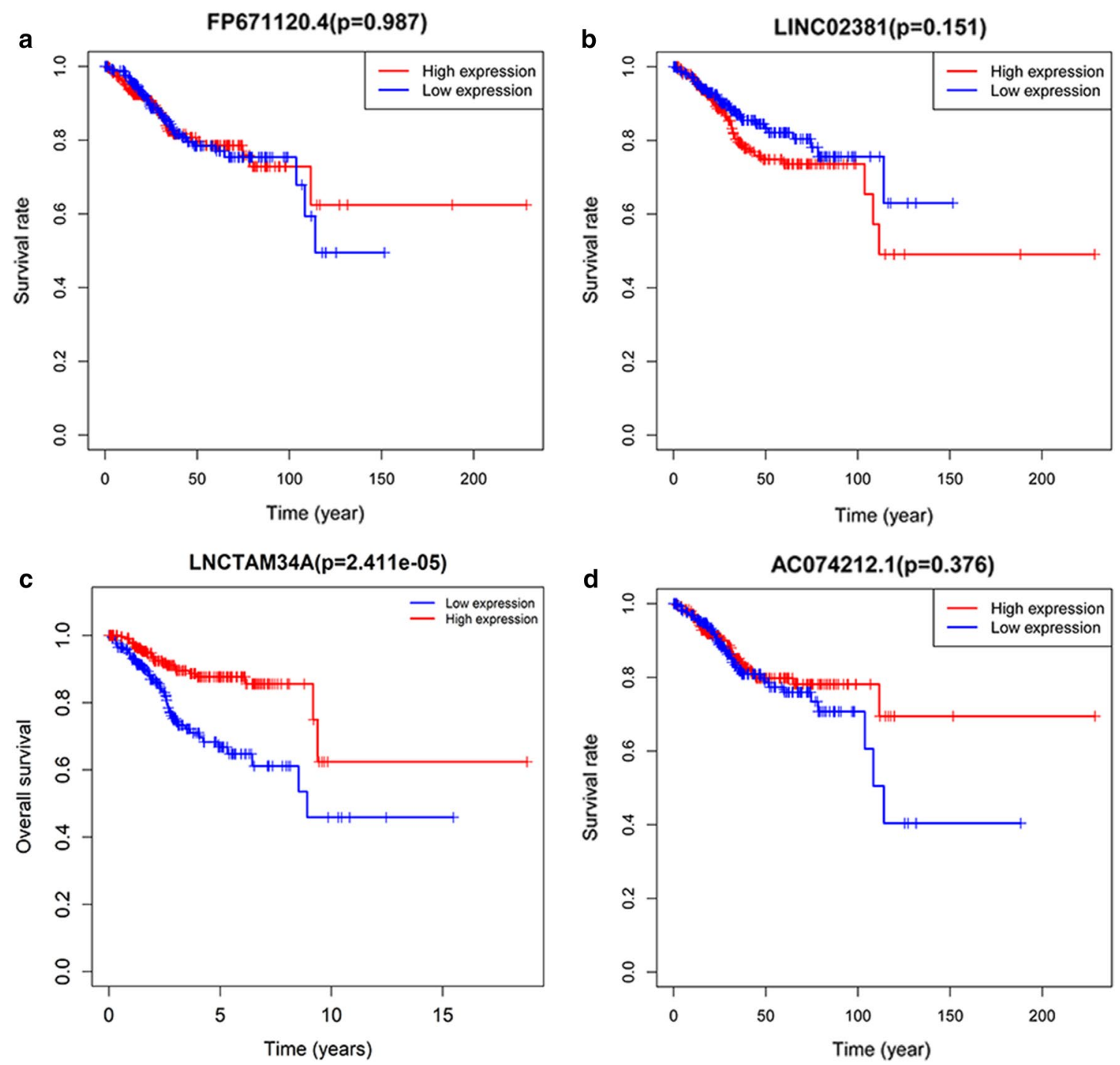

Fig. 9 The Kaplan-Meier survival of four immune-related IncRNAs respectively. a FP671120.4. b LINC02381. c LNCTAM34A. d AC074212.1

LncRNAs have been confirmed to play a key role in numerous pathological courses and remain stable in blood circulation, hence, lncRNAs are deemed biomarkers, offering guidance for therapy and determining eventual results in various carcinomas [30]. Although the abnormal expression of several lncRNAs in endometrial and ovarian cancer has been revealed in prior studies, realizing a comprehensive probe on this topic in gynecological cancers still needs long way off [31]. Pan et al. pointed out a two-way feedback ring composed of LINC01016 and miRNA that mediated cell biology phenotype transformation in EC [32]. Simultaneously, various lncRNAs have been indicated to participate in adjusting the immune system [33]. The immune response exerts a great impact on the pathophysiology and progression of solid tumors, including endometrial carcinoma. Likewise, immunotherapy, an innovative therapy modality with great promise, has recently been reported as a research hotspot [34]. Moreover, the immunoregulatory mechanism determines the occurrence and intensity of the immune response, and many transcription factors act as the main regulators to regulate the immune response process. Zaiss discussed the core role undertaken by Forkhead box transcription factors (FTFs) in the regulation of immune responses and homeostasis [19]. It is also worth noting that transcription factors and IncRNA can interact to promote appropriate regulation of gene expression [35, 36]. For instance, transcription factor SOX2 activates LINC01561 and promotes the proliferation by modulating SHCBP1 in NSCLC [37]. 

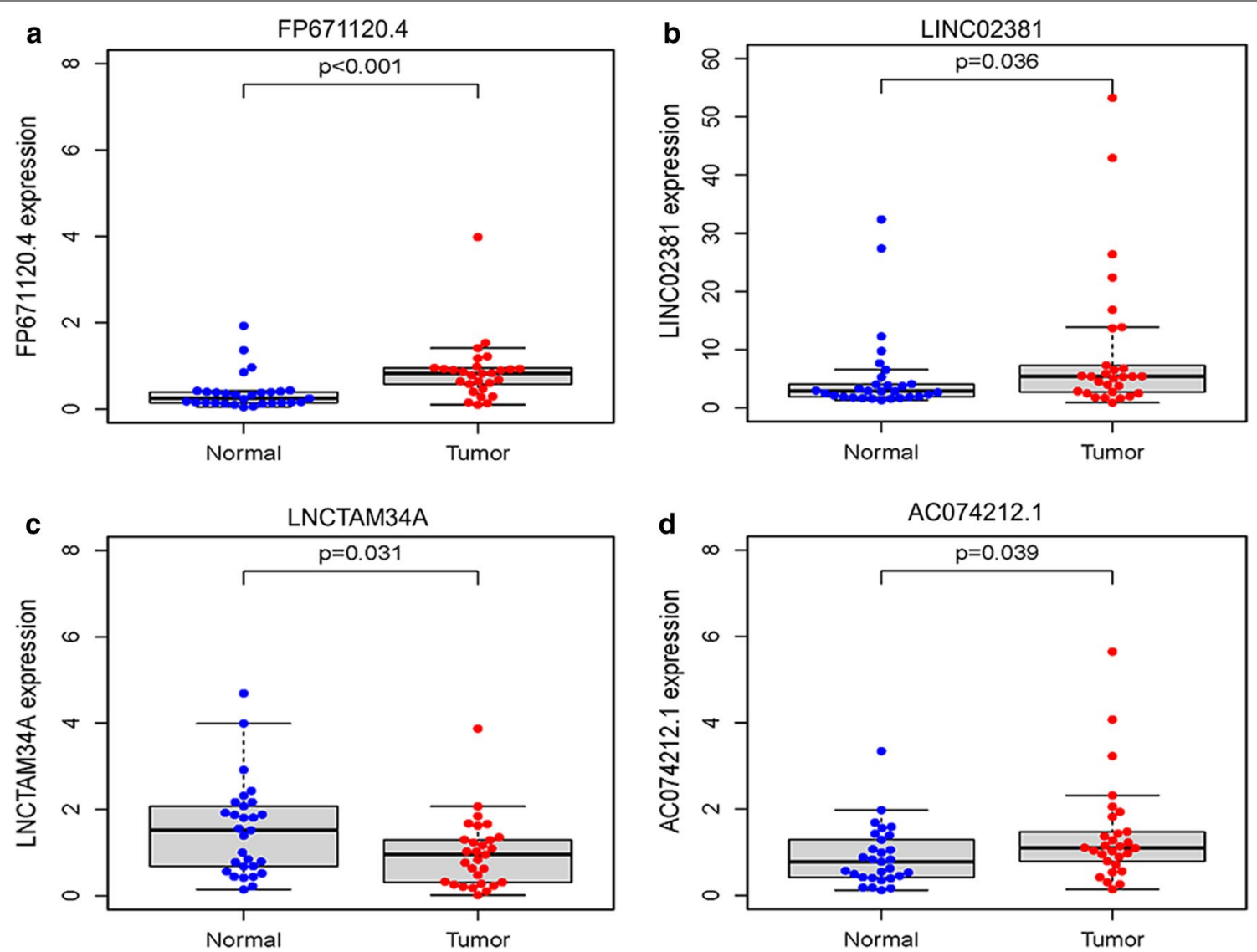

Fig. 10 QRT-PCR validation of immune-related IncRNA expression between EC tissues and normal tissues of 29 patients. a FP671120.4. b LINC02381. c LNCTAM34A. d AC074212.1

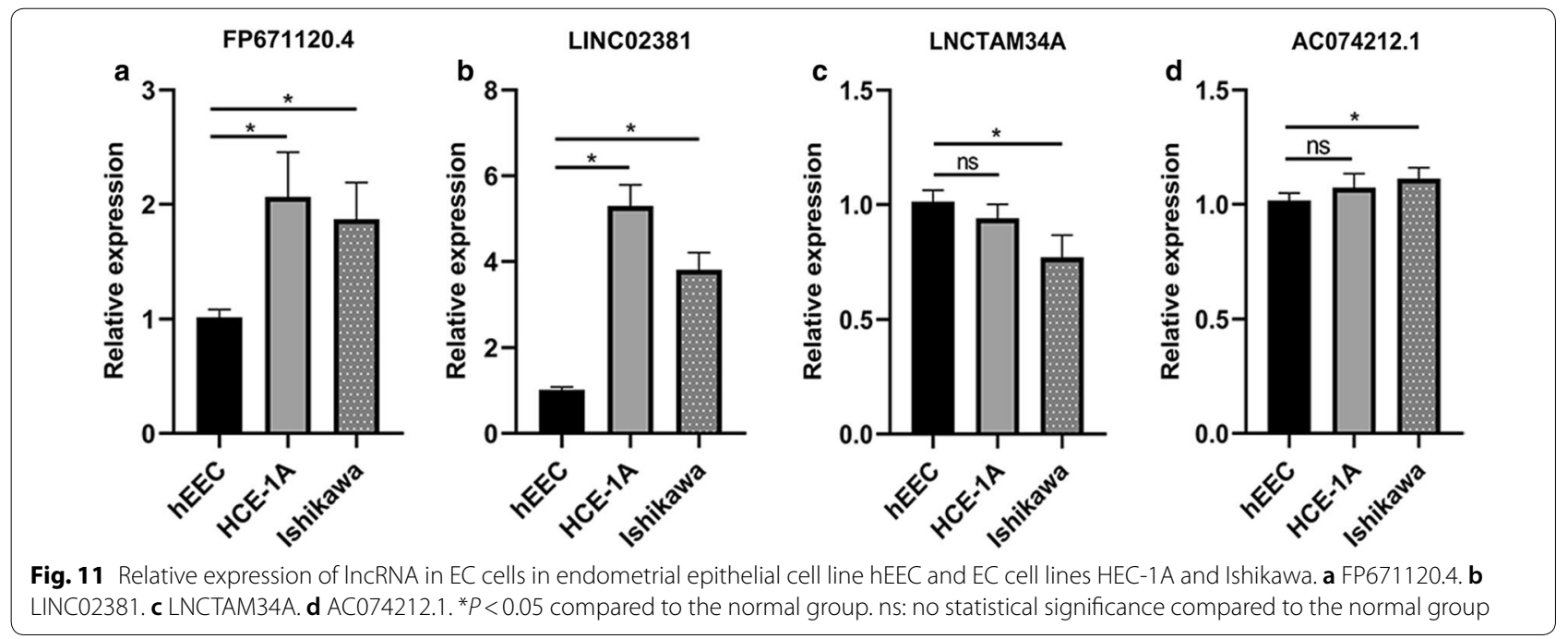

LncRNA FLICR negatively regulates transcription factor Foxp3, and the modulation may be associated with infections or tumors both related with increased Treg activity [38]. Meanwhile, the role of transcription factors in the regulation of inflammation has been increasingly reported. NF-kappa B controls the expression of multiple 


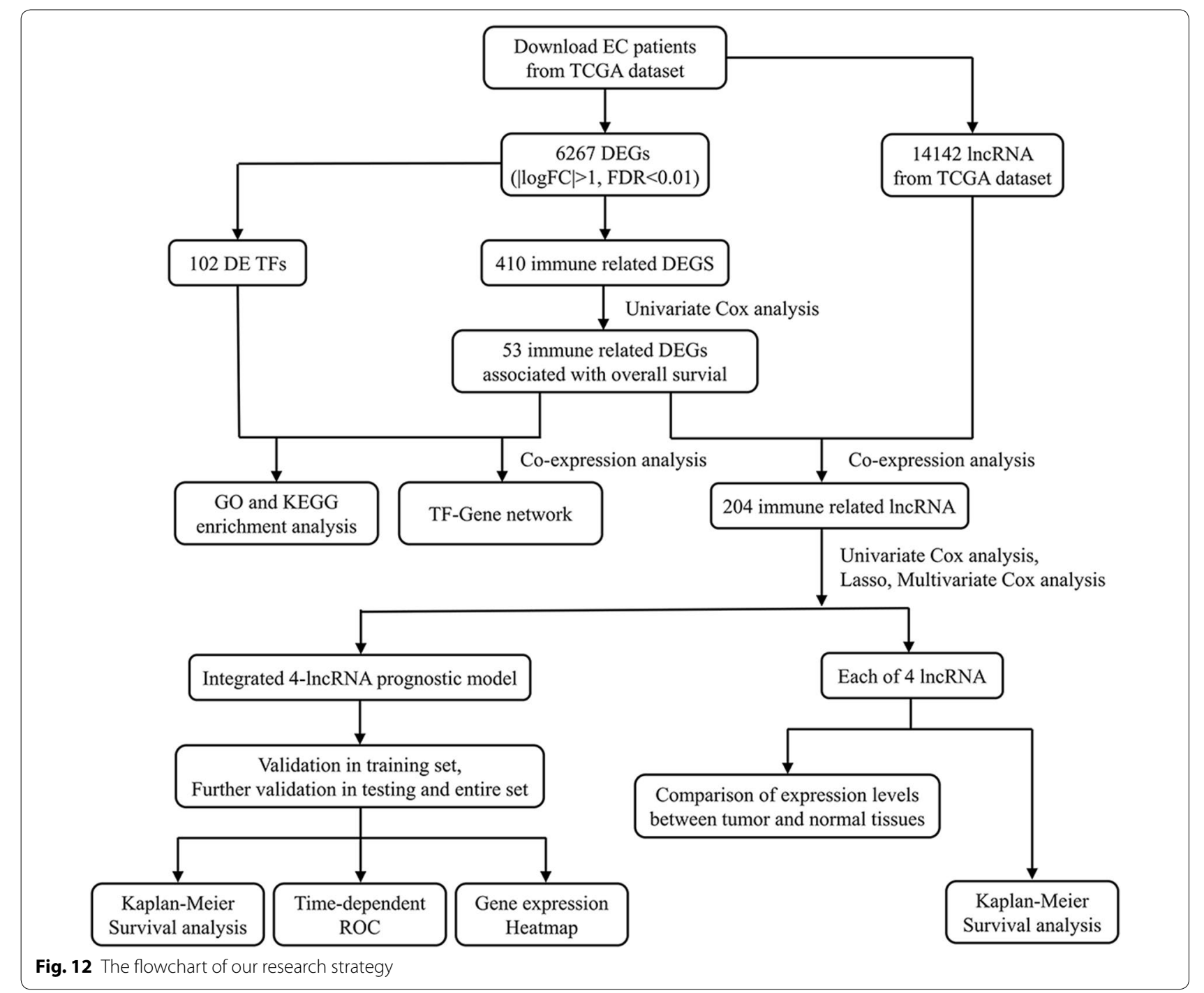

genes in endothelial cells at the site of inflammatory response, thus involving in infection and inflammation [39]. And Delpoux proposed Foxo1 has a key function in $\mathrm{T}$ cell differentiation and transport, thereby controlling the response of central memory CD8 T cells to infection [40]. In addition, years of research have adequately established the immense modulatory potential of transcription factor activity as a trigger of cancer, such as the transcription factors SNAIL1 and ZEB1, mediating the epithelialto-mesenchymal transition-related signaling pathway [41]. Kingwell et al. concluded that elF4F promotes the expression capacity of the transcription factor of STAT1, thus potentiating the immune escape of melanoma [42].

In this study, we constructed a network in EC by making use of the TCGA database to explore the interactions between DE TFs and immune-OS-related genes. There were 6267 differentially expressed genes between tumor tissues and normal tissues obtained from the TCGA.
Afterwards, the network of 29 DE TFs and 17 immuneOS-related genes was established on the basis of the results from the coexpression analysis. Furthermore, by conducting enrichment analyses, we explored $102 \mathrm{DE}$ TFs and $53 \mathrm{DE}$ genes associated with OS and the immune system, which were both included in the $6267 \mathrm{DE}$ genes. The results of GO functional analysis and KEGG enrichment analysis revealed that the DE genes are significantly clustered in the transcription factor complex, DNA-binding transcription activator activity, intracellular receptor signaling pathway, MAPK signaling pathway, PI3K-AKT signaling pathway, and PD-L1 and PD-1 checkpoint pathway, which also accounted for a major portion of the enrichment characteristics. Among them, the PTEN/ $\mathrm{PI} 3 \mathrm{~K} / \mathrm{AKT} / \mathrm{mTOR}$ pathway is the main signaling pathway participating in the metastasis of EC [43].

Moreover, we detected the association between 204 immune-related lncRNAs and EC patient survival 
prognosis by performing a series of analysis processes, such as univariate, LASSO and multivariate Cox analyses. The 4 lncRNAs, FP671120.4, LINC02381, LNCTAM34A and AC074212.1, showed notable prognostic value for EC patients in the training dataset. Next, the 4 immune-relevant lncRNAs were integrated by adopting risk scoring methods, and the results suggested that the signature could forecast patient survival independently. Furthermore, we verified the accuracy of the signature's prognostic value in the testing dataset and the entire dataset, and the results demonstrated that the development of the 4-IncRNA signature model was successful in both high-level robustness and improved repeatability in two key aspects. Our research showed that the immune score is closely linked with adverse outcomes in patients diagnosed with endometrial cancer. In addition, in accordance with univariate and multivariate analyses results, the 4-lncRNA signature was further proven to serve as an independent predictor of endometrial cancer survival prognosis. Compared with the age and grade curve, the four lncRNA signature ROC curve displayed a greater AUC. Therefore, we infer that it is reasonable to consider immune-related lncRNA that is independent of other traditional clinical features as beneficial in terms of the accuracy of measuring the prognosis of EC patients. Previous studies have constructed other prognostic models of endometrial cancer [25-28]. By comparing these models, we confirmed the prognostic ability of our immune-related lncRNA signature was higher than that of previous gene signature. It indicated that our four-lncRNA signature was better indicator for making prognosis assessment of endometrial cancer. In addition, we quantitatively analyzed these four lncRNAs by conventional qRT-PCR, and the results were consistent with the above-mentioned bioinformatics results. Therefore, the biological signature we constructed can be identified as a valuable and reliable predictor.

In subsequent steps, to provide better application to clinical diagnosis, we estimated the expression differences of individual lncRNAs among the 4-lncRNA signature between carcinoma tissues and normal tissues and performed KM survival analysis for each of the four lncRNAs. The results showed that patients with high LNCTAM34A expression had a longer survival time than those with low expression, while there was no significant difference in LNCTAM34A expression between cancer and normal tissues, perhaps because the data sample we used was limited. In addition, LNCTAM34A has been found to mediate the high expression of miR34a to protect cells from outside stress stimuli [44]. Moreover, the expression results of two genes in PCR were not identical to each of those analyzed in database. It may be due to the small sample size of normal endometrial tissue in the database or heterogeneity of the tumor.

Nevertheless, there remain certain limitations to our study. To solve the problem of insufficient samples, our signature should be adequately validated in other databases and in studies with larger amounts of endometrial cancer data. The major research analysis approach we employed is bioinformatics technology, which has emerged as an effective and reliable tool, while the functional mechanism and interaction network of lncRNAs are complicated. Further functional studies on the lncRNAs we explored, the acquisition of additional experimental data (in vitro and in vivo) and long-term follow-up observations are essential to estimate the accuracy of our signature and confirm our findings.

\section{Conclusions}

In summary, our team proposed a 4-IncRNA signature based on the immune system as a possible underlying biomarker for effective diagnosis and prognosis assessment. This signature could be considered a novel target of an immunological approach for endometrial cancer with a bright perspective (Additional file 8: Figure S7, Additional file 9: Table S2).

\section{Supplementary information}

Supplementary information accompanies this paper at https://doi. org/10.1186/s12935-020-01572-6.

Additional file 1: Figure S1. The calculation formula of risk score. Additional file 2: Figure S2. Statement on informed consent.

Additional file 3: Table S1. Primers applied in qPCR.

Additional file 4: Figure S3. Identification of immune related and differentially expressed genes associated with overall survival (OS) of EC patients. The forest plot showed 53 out of 410 immune-related DE genes are related to survival.

Additional file 5: Table S2. Co-expression analysis of DE TFs and immune-OS-related DE genes. The co-expression analysis result displays that there were interactions between 29 DE TFs and 17 immune-OSrelated DE genes.

Additional file 6: Figure S4. Verification of the signature in the testing set. (a) Kaplan-Meier survival analysis between high- and low-risk groups patients with EC. (b) Receiver operating characteristic (ROC). (c) The distribution of risk score, survival duration and expression profiles of 4-IncRNA in high- and low-risk groups.

Additional file 7: Figure S5. Verification of the signature in the entire set. (a) Kaplan-Meier survival analysis between high- and low-risk groups patients with EC. (b) Receiver operating characteristic (ROC). (c) The distribution of risk score, survival duration and expression profiles of 4-IncRNA in high- and low-risk groups.

Additional file 8: Figure S6. Assessment of independent risk factors in training set. (a) Age, grade and risk score were the independent prognostic indicators by univariate analysis. (b) Grade and risk score were the independent prognostic indicators by multivariate analysis. (c) ROC curves showed the predict potential of 4-IncRNA signature. 
Additional file 9: Figure S7. Assessment of independent risk factors in testing set. (a) Grade and risk score were the independent prognostic indicators by univariate analysis. (b) Grade and risk score were the independent prognostic indicators by multivariate analysis. (c) ROC curves showed the predict potential of 4-IncRNA signature.

\section{Abbreviations}

EC: Endometrial cancer; ROC: Receiver operating characteristic; TCGA: The Cancer Genome Atlas; DE: Differential expression; TF: Transcription factor; DEG: Differentially expressed gene; OS: Overall survival; PCA: Principal component analysis.

\section{Acknowledgements}

None.

\section{Authors' contributions}

$X C$ and $H Y$ designed the study, $H Y, Y W$ and $Y L$ performed the research, analysed the data and wrote the manuscript, YG, HL, YL, MZ, PC and DK contributed to carrying out additional analyses, discussed the results, provided scientific advice and revised the manuscript. All authors read and approved the final manuscript.

\section{Funding}

This work was supported by the National Natural Science Fund of China (No. 81903120 to H. Yin, No. 81772274 to X.W. Chen), and Nn10 plan project (Nn10py2017-01 to X.W. Chen).

\section{Data availability statement}

The data supporting the findings of this study are available from the corresponding author upon request.

\section{Ethics approval and consent to participate}

The whole study was approved by the Ethics Committee of Harbin Medical University.

\section{Consent for publication}

Not applicable.

\section{Competing interests}

There are no conflicts of interest to be reported.

Received: 29 July 2020 Accepted: 23 September 2020

Published online: 02 October 2020

\section{References}

1. Byrne FL, Poon IK, Modesitt SC, Tomsig JL, Chow JD, Healy ME, Baker WD, Atkins KA, Lancaster JM, Marchion DC, et al. Metabolic vulnerabilities in endometrial cancer. Can Res. 2014;74(20):5832-45.

2. Soumerai TE, Donoghue MTA, Bandlamudi C, Srinivasan P, Chang MT, Zamarin D, Cadoo KA, Grisham RN, O'Cearbhaill RE, Tew WP, et al. Clinical Utility of Prospective Molecular Characterization in Advanced Endometrial Cancer. Clin Cancer Res. 2018;24(23):5939-47.

3. Bruegl AS, Ring KL, Daniels M, Fellman BM, Urbauer DL, Broaddus RR. Clinical challenges associated with universal screening for lynch syndrome-associated endometrial cancer. Cancer Prev Res (Philadelphia, Pa). 2017;10(2):108-15.

4. Bian X, Gao J, Luo F, Rui C, Zheng T, Wang D, Wang Y, Roberts TM, Liu P, Zhao JJ, et al. PTEN deficiency sensitizes endometrioid endometrial cancer to compound PARP-PI3K inhibition but not PARP inhibition as monotherapy. Oncogene. 2018;37(3):341-51.

5. Zhao Y, Yang Y, Trovik J, Sun K, Zhou L, Jiang P, Lau TS, Hoivik EA, Salvesen $\mathrm{HB}$, Sun $\mathrm{H}$, et al. A novel wnt regulatory axis in endometrioid endometrial cancer. Can Res. 2014;74(18):5103-17.
6. Haines K, Huang GS. Precision therapy for aggressive endometrial cancer by reactivation of protein phosphatase $2 \mathrm{~A}$. Can Res. 2019;79(16):4009-100.

7. Kim J, Piao HL, Kim BJ, Yao F, Han Z, Wang Y, Xiao Z, Siverly AN, Lawhon SE, Ton BN, et al. Long noncoding RNA MALAT1 suppresses breast cancer metastasis. Nat Genet. 2018;50(12):1705-15.

8. Lin C, Yang L. Long noncoding RNA in cancer: wiring signaling circuitry. Trends Cell Biol. 2018;28(4):287-301.

9. Wu Y, Yang $X$, Chen Z, Tian L, Jiang G, Chen F, Li J, An P, Lu L, Luo N, et al m(6)A-induced IncRNA RP11 triggers the dissemination of colorectal cancer cells via upregulation of Zeb1. Mol Cancer. 2019;18(1):87.

10. Zhou M, Zhao H, Xu W, Bao S, Cheng L, Sun J. Discovery and validation of immune-associated long non-coding RNA biomarkers associated with clinically molecular subtype and prognosis in diffuse large B cell lymphoma. Mol Cancer. 2017;16(1):16.

11. Xu Q, Yin H, Ao H, Leng X, Liu M, Liu Y, Ma J, Wang X. An 11-IncRNA expression could be potential prognostic biomarkers in head and neck squamous cell carcinoma. J Cell Biochem. 2019;120(10):18094-103.

12. Yin $H$, Wang $X$, Zhang $X$, Wang $Y$, Zeng $Y$, Xiong $Y$, Li T, Lin R, Zhou Q, Ling $H$, et al. Integrated analysis of long noncoding RNA associatedcompeting endogenous RNA as prognostic biomarkers in clear cell renal carcinoma. Cancer Sci. 2018;109(10):3336-49.

13. Keren L, Bosse M, Marquez D, Angoshtari R, Jain S, Varma S, Yang SR, Kurian A, Van Valen D, West $R$, et al. A structured tumor-immune microenvironment in triple negative breast cancer revealed by multiplexed ion beam imaging. Cell. 2018;174(6):1373-1387.e1319.

14. Ferriss JS, Williams-Brown MY. Immunotherapy: checkpoint inhibitors in lynch-associated gynecologic cancers. Curr Treat Options Oncol. 2019;20(10):75.

15. Marinelli O, Annibali D, Aguzzi C, Tuyaerts S, Amant F, Morelli MB, Santoni G, Amantini C, Maggi F, Nabissi M. The controversial role of PD-1 and its ligands in gynecological malignancies. Front Oncol. 2019;9:1073.

16. Chen YG, Satpathy AT, Chang HY. Gene regulation in the immune system by long noncoding RNAs. Nat Immunol. 2017;18(9):962-72.

17. Atianand MK, Caffrey DR, Fitzgerald KA. Immunobiology of long noncoding RNAs. Annu Rev Immunol. 2017;35:177-98.

18. Igarashi K, Kurosaki T, Roychoudhuri R. BACH transcription factors in innate and adaptive immunity. Nat Rev Immunol. 2017;17(7):437-50.

19. Zaiss DMW, Coffer PJ. Forkhead box transcription factors as contextdependent regulators of lymphocyte homeostasis. Nat Rev Immunol. 2018;18(11):703-15.

20. Liu T, Ortiz JA, Taing L, Meyer CA, Lee B, Zhang Y, Shin H, Wong SS, Ma J, Lei $Y$, et al. Cistrome: an integrative platform for transcriptional regulation studies. Genome Biol. 2011;12(8):R83.

21. Zalocusky KA, Kan MJ, Hu Z, Dunn P, Thomson E, Wiser J, Bhattacharya S, Butte AJ. The 10,000 Immunomes project: building a resource for human immunology. Cell Rep. 2018;25(2):513-522.e513.

22. Shannon P, Markiel A, Ozier O, Baliga NS, Wang JT, Ramage D, Amin N, Schwikowski B, Ideker T. Cytoscape: a software environment for integrated models of biomolecular interaction networks. Genome Res. 2003;13(11):2498-504.

23. Wang $X$, Yin H, Zhang H, Hu J, Lu H, Li C, Cao M, Yan S, Cai L. NF-kBdriven improvement of EHD1 contributes to erlotinib resistance in EGFR-mutant lung cancers. Cell Death Dis. 2018;9(4):418.

24. Yin H, Ma J, Chen L, Piao S, Zhang Y, Zhang S, Ma H, Li Y, Qu Y, Wang $X$, et al. MiR-99a Enhances the Radiation Sensitivity of NonSmall Cell Lung Cancer by Targeting mTOR. Cell Physiol Biochem. 2018;46(2):471-81.

25. O'Mara TA, Zhao M, Spurdle AB. Meta-analysis of gene expression studies in endometrial cancer identifies gene expression profiles associated with aggressive disease and patient outcome. Sci Rep. 2016;6:36677.

26. Wang Y, Ren F, Chen P, Liu S, Song Z, Ma X. Identification of a six-gene signature with prognostic value for patients with endometrial carcinoma. Cancer Med. 2018;7(11):5632-42.

27. Liu L, Lin J, He H. Identification of potential crucial genes associated with the pathogenesis and prognosis of endometrial cancer. Front Genet. 2019;10:373. 
28. Wang ZH, Zhang YZ, Wang YS, Ma XX. Identification of novel cell glycolysis related gene signature predicting survival in patients with endometrial cancer. Cancer Cell Int. 2019;19:296.

29. Brooks RA, Fleming GF, Lastra RR, Lee NK, Moroney JW, Son CH, Tatebe K, Veneris $\mathrm{JL}$. Current recommendations and recent progress in endometrial cancer. CA a Cancer J Clin. 2019;69(4):258-79.

30. Chen X, Dai M, Zhu H, Li J, Huang Z, Liu X, Huang Y, Chen J, Dai S. Evaluation on the diagnostic and prognostic values of long non-coding RNA BLACAT1 in common types of human cancer. Mol Cancer. 2017;16(1):160.

31. Hosseini ES, Meryet-Figuiere M, Sabzalipoor H, Kashani HH, Nikzad H, Asemi Z. Dysregulated expression of long noncoding RNAs in gynecologic cancers. Mol Cancer. 2017;16(1):107.

32. Pan X, Li D, Huo J, Kong F, Yang H, Ma X. LINC01016 promotes the malignant phenotype of endometrial cancer cells by regulating the miR302a-3p/miR-3130-3p/NFYA/SATB1 axis. Cell Death Dis. 2018;9(3):303.

33. Heward JA, Lindsay MA. Long non-coding RNAs in the regulation of the immune response. Trends Immunol. 2014;35(9):408-19.

34. Yang J, Hu L. Immunomodulators targeting the PD-1/PD-L1 proteinprotein interaction: from antibodies to small molecules. Med Res Rev. 2018;39:265-301

35. Holmes ZE, Hamilton DJ, Hwang T, Parsonnet NV, Rinn JL, Wuttke DS, Batey RT. The Sox 2 transcription factor binds RNA. Nat Commun. 2020;11(1):1805.

36. Wang K, Ye X, Yang C, Chen G, Yao N, Kang Z, Shi W. Comprehensive analysis of novel IncRNA-TF regulatory cross talks and identification of core IncRNA-TF feedback loops in sarcoma. DNA Cell Biol. 2020. https:// doi.org/10.1089/dna.2020.5385.

37. Gao W, Qi CQ, Feng MG, Yang P, Liu L, Sun SH. SOX2-induced upregulation of IncRNA LINC01561 promotes non-small-cell lung carcinoma progression by sponging miR-760 to modulate SHCBP1 expression. J Cell Physiol. 2020;235(10):6684-96.
38. Zemmour D, Pratama A, Loughhead SM, Mathis D, Benoist C. Flicr, a long noncoding RNA, modulates Foxp3 expression and autoimmunity. Proc Natl Acad Sci USA. 2017;114(17):E3472-e34803480.

39. Bianco C, Thompson L, Mohr I. Repression of eEF2K transcription by $\mathrm{NF}-\mathrm{kB}$ tunes translation elongation to inflammation and dsDNA-sensing. Proc Natl Acad Sci USA. 2019;116(45):22583-90.

40. Delpoux A, Michelini RH, Verma S, Lai CY, Omilusik KD, Utzschneider DT, Redwood AJ, Goldrath AW, Benedict CA, Hedrick SM. Continuous activity of Foxo1 is required to prevent anergy and maintain the memory state of CD8(+) T cells. J Exp Med. 2018;215(2):575-94.

41. Bushweller JH. Targeting transcription factors in cancer - from undruggable to reality. Nat Rev Cancer. 2019;19(11):611-24.

42. Kingwell K. Anticancer drugs: translational target for checkpoint inhibitors. Nat Rev Drug Discov. 2018;17(12):863.

43. Dong P, Xiong Y, Yue J, Hanley SJB, Kobayashi N, Todo Y, Watari H. Exploring IncRNA-mediated regulatory networks in endometrial cancer cells and the tumor microenvironment: advances and challenges. Cancers. 2019;11(2):234

44. Serviss JT, Andrews N, Van den Eynden J, Richter FC, Houtman M, Vesterlund M, Schwarzmueller L, Johnsson P, Larsson E, Grander D, et al. An antisense RNA capable of modulating the expression of the tumor suppressor microRNA-34a. Cell Death Dis. 2018;9(7):736.

\section{Publisher's Note}

Springer Nature remains neutral with regard to jurisdictional claims in published maps and institutional affiliations.

Ready to submit your research? Choose BMC and benefit from:

- fast, convenient online submission

- thorough peer review by experienced researchers in your field

- rapid publication on acceptance

- support for research data, including large and complex data types

- gold Open Access which fosters wider collaboration and increased citations

- maximum visibility for your research: over $100 \mathrm{M}$ website views per year

At $B M C$, research is always in progress.

Learn more biomedcentral.com/submissions 